\title{
Determinación de la ploidía en accesiones de papas nativas (Solanum tuberosum grupo Andigenum) procedentes del Banco de Germoplasma vegetal de CORPOICA
}

\author{
Determination of ploidy in native potato accessions (Solanum tuberosum group \\ Andigenum) from the Plant Germplasm Bank of CORPOICA
}

\section{Determinação da ploidia em acessos de batata nativa (grupo de Solanum tuberosum Andigenum) do Banco de Germoplasma de Plantas de CORPOICA}

\author{
Manuel Alejandro Sánchez ${ }^{1}$
}

Forma de citar: M.A. Sánchez, "Determinación de la ploidía en accesiones de papas nativas (Solanum tuberosum grupo Andigenum) procedentes del Banco de Germoplasma vegetal de CORPOICA", Respuestas, vol. 22, no. 2, pp. 6-13, 2017.

\section{Recibido:}

Noviembre 16 de 2016

Aceptado:

Abril 28 de 2017

\section{Resumen}

Antecedentes. Las colecciones de germoplasma buscan ampliar la base genética para que los mejoradores dispongan de genotipos deseables. En Colombia hay gran diversidad de papas (Solanum tuberosum L.), que son conservadas y usadas en el mejoramiento genético del cultivo. Es importante contar con mayor información de las accesiones, como los niveles de ploidía, lo que permitiría reforzar las estrategias de uso y aprovechamiento de la diversidad genética en esquemas de pre-mejoramiento. Objetivo. Determinar la ploidía de 30 accesiones de S. tuberosum grupo Andigenum, del Banco de Germoplasma, administrado por CORPOICA. Método. Se realizó el conteo de cloroplastos en células guarda y conteo de cromosomas en células somáticas de punta de raíz, siguiendo procedimientos convencionales. Resultados. Se identificaron tres accesiones diploides $(2 \mathrm{n}=2 \mathrm{x}=24)$, que a la vez, presentaron 7 a 8 cloroplastos en las células guarda, el resto de accesiones fueron tetraploides $(2 n=4 x=48)$ que a su vez, presentaron 12 a 14 cloroplastos en sus células guarda. Conclusión. Se encontró correspondencia entre el número de cromosomas y el número de cloroplastos, lo cual permitió verificar dos niveles de ploidía en los materiales estudiados, todos correspondientes a S. tuberosum grupo Andigenum.

Palabras clave: Citogenética, Cloroplastos, Cromosomas, Papa.
${ }^{1}$ Ingeniero Biotecnológico manuel.27116218240@ucaldas. edu.co. Orcid: 0000-0002-2970-8825. Universidad de Caldas. Manizales, Colombia

\begin{abstract}
Background. Germplasm collections seek to broaden the genetic base for breeders to have desirable genotypes. In Colombia there is a great diversity of potatoes (Solanum tuberosum L.), which are conserved and used in the genetic improvement of the crop. For this reason it is important to know the genotype of the accessions by cytogenetic techniques; which allows to reinforce the taxonomic identification to be used in schemes of pre-improvement. Objective. To determine the ploidy of 30 accessions of S. tuberosum group andigenum, from the Germplasm Bank, administered by Corpoica. Method. Chloroplasts were counted in guard cells and counts of chromosomes in somatic cells of root tip, following conventional procedures. Results. Three diploid accessions $(2 n=2 x$
\end{abstract}


$=24$ ) were identified, which at the same time presented 7 to 8 chloroplasts in the guard cells, the remaining accessions were tetraploids $(2 \mathrm{n}=4 \mathrm{x}=48)$, which in turn presented 12 to 14 Chloroplasts in their guard cells. Conclusion. Correspondence was found between the number of chromosomes and the number of chloroplasts. This allowed to verify two ploidy levels in the materials under study, all belonging to S. tuberosum group Andigenum.

Keywords: chloroplasts, chromosomes, cytogenetic, potato.

\section{Resumo}

Background. coleções de germoplasma buscam ampliar a base genética disponível para criadores de genótipos desejáveis. Em Colômbia existe uma grande diversidade de batata (Solanum tuberosum L.), que são preservados e utilizados na reprodução de culturas. É importante ter mais informações sobre os acessos, tais como níveis de ploidia, que iria fortalecer estratégias de utilização e exploração da diversidade genética em esquemas pré-aperfeiçoamento. Objetivo. Determinar a ploidia de 30 acessos de grupo de S. tuberosum andigenum, a Genebank, CORPOICA administrado. Método. contando cloroplastos em células guarda e foi realizada cromossoma contagem de células somáticas no ponta da raiz, seguindo procedimentos padrão. Resultados. três acessos diplóides ( $2 \mathrm{n}$ $=2 \mathrm{x}=24$ ), o qual, por sua vez, teve foram identificadas 7 a 8 cloroplastos em células guarda, outros acessos foram tetraplóide $(2 \mathrm{n}=4 \mathrm{x}=48)$ que, por sua vez, apresentaram 12 a 14 cloroplastos em suas células salvo. Conclusão. correspondência entre o número de cromossomas e o número de cloroplastos encontrado, o que permitiu verificar dois níveis de ploidia nos materiais estudados, todos tuberosum grupo correspondente $\mathrm{S}$. andigenum.

Palavras-chave: cloroplastos, cromossomos, citogenética, papa.

\section{Introducción}

La papa (Solanum tuberosum L.) es uno de los cultivos alimenticios básicos para la población del mundo, ocupa el cuarto lugar en producción mundial después del trigo, el maíz y el arroz [1]. Satisface parte importante de los requerimientos energéticos y nutricionales en diferentes países [2]. En Colombia, la papa se cultiva en una superficie de 125.600 hectáreas/año, con una producción de 2.5 millones de toneladas [3]. Los principales departamentos productores con $68 \%$ de la superficie cosechada son Cundinamarca y Boyacá, seguido por Nariño y Antioquia con $20 \%$ y el $12 \%$ entre otros [3].

Si bien, Colombia no es centro de origen de la papa (S. tuberosum), el país cuenta con amplia diversidad genética en especies silvestres y cultivadas [4]. Dada la gran variabilidad de esta especie, los programas de mejoramiento genético necesitan conservar y manejar numerosos genotipos en las colecciones de germoplasma, los cuales, más adelante, pueden ser utilizados como progenitores [5]. La mayoría de estos genotipos son portadores de genes valiosos, relacionados con producción y resistencia a estrés bióticos y abióticos que a través de cruzamientos son transferidos a nuevas variedades mejoradas [6]. Parte de la información genética de estos materiales puede ser obtenida mediante técnicas citogenéticas y moleculares basadas en pruebas de ADN, con ellas se han identificado clones, y cultivares de papa que facilitan enormemente el uso del germoplasma [7].
Julio - Dic. 2017

ISSN 0122-820X

E-ISSN 2422-5053

PP: 6-13 
Respuestas

Cúcuta-Colombia

Vol. 22

No. 2

Julio - Dic. 2017

ISSN 0122-820X

E-ISSN 2422-5053

PP: 6-13
Determinación de la ploidía en accesiones de papas nativas (Solanum tuberosum grupo Andigenum) procedentes del Banco de Germoplasma vegetal de CORPOICA
La colección del germoplasma de papa en Colombia se encuentra en custodía por Corpoica-Tibaitatá y está constituida por aproximadamente 1.980 accesiones. Este germoplasma se ha evaluado a nivel morfológico en años anteriores y son mantenidos bajo condiciones de campo. El tamaño de esta colección está en constante cambio y regularmente se integran nuevos genotipos, los cuales provienen de selecciones avanzadas del mismo programa $\mathrm{y}$ de introducciones de materiales de otros programas y regiones [8].

Dicho lo anterior se hace necesario complementar la información de la colección conociendo el nivel de ploidia de estos materiales, con el fin de aprovechar mejor los recursos genéticos que posee la colección. En este sentido, el objetivo de la presente investigación fue determinar la ploidía de un selecto grupo de 30 accesiones de papa nativas (Solanum tuberosum L.) mediante conteo cromosómico y conteo de cloroplastos en células guarda. Los resultados aportan información básica útil para el mejoramiento genético de la especie. Además, contribuye con nuevo conocimiento sobre las accesiones y la actualización de la base de datos de la Colección Central Colombiana de Papa perteneciente a CORPOICA.

\section{Materiales y Métodos}

\subsection{Material vegetal}

Se usaron 30 accesiones de la Colección Central de Papa del Banco de Germoplasma de CORPOICA (Tabla I), crecidos en turba canadiense bajo condición de invernadero, con temperatura promedio de $18^{\circ} \mathrm{C}$. Estos materiales han sido evaluados en años anteriores de manera parcial, con mayor énfasis en la caracterización morfoagronómica, representando así la máxima diversidad en cuanto a su origen geográfico y constituye el grupo de progenitores más frecuentemente utilizados en los cruzamientos que se realizan en los programas de mejoramiento.

Tabla I. Accesiones de trabajo: Código asignado en la colección de CORPOICA, nombre común, procedencia del material y clasificación taxonómica.

\begin{tabular}{|c|c|c|c|}
\hline $\begin{array}{l}\text { CÓDIGO DE } \\
\text { COLECCIÓN }\end{array}$ & NOMBRE COMÚN & PROCEDENCIA & $\begin{array}{c}\text { CLASIFICACIÓN } \\
\text { TAXONOMÍCA } \\
\text { PRELIMINAR }\end{array}$ \\
\hline 061291 & Pana rosada & Cundinamarca & *Andigenum \\
\hline 061641 & Lizarasa & Cundinamarca & Andigenum \\
\hline 061951 & Curipamba Blanca & Boyacá & Andigenum \\
\hline 062107 & Curipamba & Nariño & Andigenum \\
\hline 061300 & Carrizo & Nariño & Andigenum \\
\hline 061273 & Argentina parda & Cundinamarca & Andigenum \\
\hline 061777 & Bola de Sal & Cundinamarca & Andigenum \\
\hline 061932 & Arbolona Blanca & Boyacá & Andigenum \\
\hline 061286 & Argentina & Boyacá & Andigenum \\
\hline 060188 & Carrizo & Nariño & Andigenum \\
\hline 061336 & Lizaraza Grande & Cundinamarca & Andigenum \\
\hline 061327 & Pana & Cundinamarca & Andigenum \\
\hline 062039 & Mampuera & Cundinamarca & Andigenum \\
\hline 062357 & Argentina & Cundinamarca & Andigenum \\
\hline 061815 & Pastusa Palinegra & Boyacá & Andigenum \\
\hline 061469 & Leona & Cundinamarca & Andigenum \\
\hline 062138 & Arbolona & Cundinamarca & Andigenum \\
\hline 061295 & Argentina Colorada & Boyacá & Andigenum \\
\hline 061439 & Pamba Blanca & Cundinamarca & Andigenum \\
\hline 061231 & Arbolona Rosada & Cundinamarca & Andigenum \\
\hline 062071 & Arbolona Pintada & Cundinamarca & Andigenum \\
\hline 061979 & Uva & Cundinamarca & Andigenum \\
\hline 061665 & Palinegra & Nariño & Andigenum \\
\hline 061445 & Variedad Colombiana & Cundinamarca & Andigenum \\
\hline 061991 & Yema de Huevo & Nariño & Andigenum \\
\hline 061434 & Curipamba Negra & Cundinamarca & Andigenum \\
\hline 061632 & Londres & Cundinamarca & Andigenum \\
\hline 062517 & Ratona & Boyacá & Andigenum \\
\hline 062407 & Tocana & Nariño & Andigenum \\
\hline 061415 & Colombia & Nariño & Andigenum \\
\hline
\end{tabular}

*Leer como Solanum tuberosum grupo Andigenum 


\subsection{Análisis citogenético}

La determinación del número cromosómico en raíz se realizó con la técnica squash o aplastamiento siguiendo el protocolo propuesto por Orrillo y Bonierbale [9], con algunas modificaciones. Los cambios consistieron en: primero, pre-fijación con agua helada lo que reemplazó el insecticida piretroide; segundo, se suprimió la fijación en Carnoy 3:1 durante 48 horas; y tercero, el ácido clorhídrico $(\mathrm{HCl} 1 \mathrm{~N})$ se usó a temperatura ambiente evitando su precalentamiento a $60^{\circ} \mathrm{C}$. De este modo se optimizaron recursos y tiempo. La preparación de las placas consistió en la colecta de puntas de raíz teniendo en cuenta la hora mitótica entre las 8:30 am y las 9:00 am en un prefijador (agua semicongelada). Las raíces fueron expuestas al prefijador por 24 horas a $4{ }^{\circ} \mathrm{C}$. Inmediatamente después las raíces se fijaron en una solución de ácido clorhídrico $(\mathrm{HCl}) 1 \mathrm{~N}$ durante media hora y se enjuagaron con agua destilada. A continuación se sumergieron en una solución de orceína lacto propiónica durante 1 hora. Las puntas de las raíces se colocaron sobre un cubreobjetos y se aplicó presión sobre las mismas. Después, con el dedo pulgar y con una pieza de tela sobre el montaje, se ejerció presión para disgregar aún más las células. El conteo de los cromosomas, se realizó con microscopio óptico en aumentos de 10x, 20x, 40x, y luego con aceite de inmersión en 100x. Se examinaron y contaron los cromosomas en 10 células metafásicas. Este proceso se repitió tres veces por cada accesión.

\subsection{Análisis citológico}

La determinación del número de cloroplastos en células guardas fue realizada según el protocolo establecido por Orrillo y Bonierbale [9]. Se tomaron de 3 a 5 hojas apicales de cada una de las accesiones, se introdujeron en una caja petri con papel absorbente y alcohol etílico al $70 \%$, se desprendió, con pinza de punta fina, la dermis del envés y se colocó en porta objeto. Se tiñó con dos gotas de solución de yoduro de potasio (KI), la cual da un color rojizo a los cloroplastos. Se colocó el cubre objeto y se secó el exceso de solución (KI). Se agregó esmalte transparente en el borde del cubre objeto y se dejó secar. Se observó en el microscopio en 10x, 20x, 40x y 100x, se contó el número de cloroplastos en 10 células guarda y se obtuvo el promedio.

\section{Resultados y análisis}

\subsection{Análisis citogenético}

De las 30 accesiones evaluadas, tres fueron diploides y 27 tetraploides, presentando 24 y 48, cromosomas respectivamente (Tabla II). Un ejemplo de lo observado en cada grupo se muestra en la Figura 1. Estos resultados coinciden con la literatura, ya que en papa cultivada existen cinco niveles de ploidía: diploide $(2 \mathrm{n}=2 \mathrm{x}=24)$, triploide $(2 \mathrm{n}=3 \mathrm{x}=$ $36)$, tetraploide $(2 \mathrm{n}=4 \mathrm{x}=48)$, pentaploide $(2 \mathrm{n}=5 \mathrm{x}=60)[10]$.
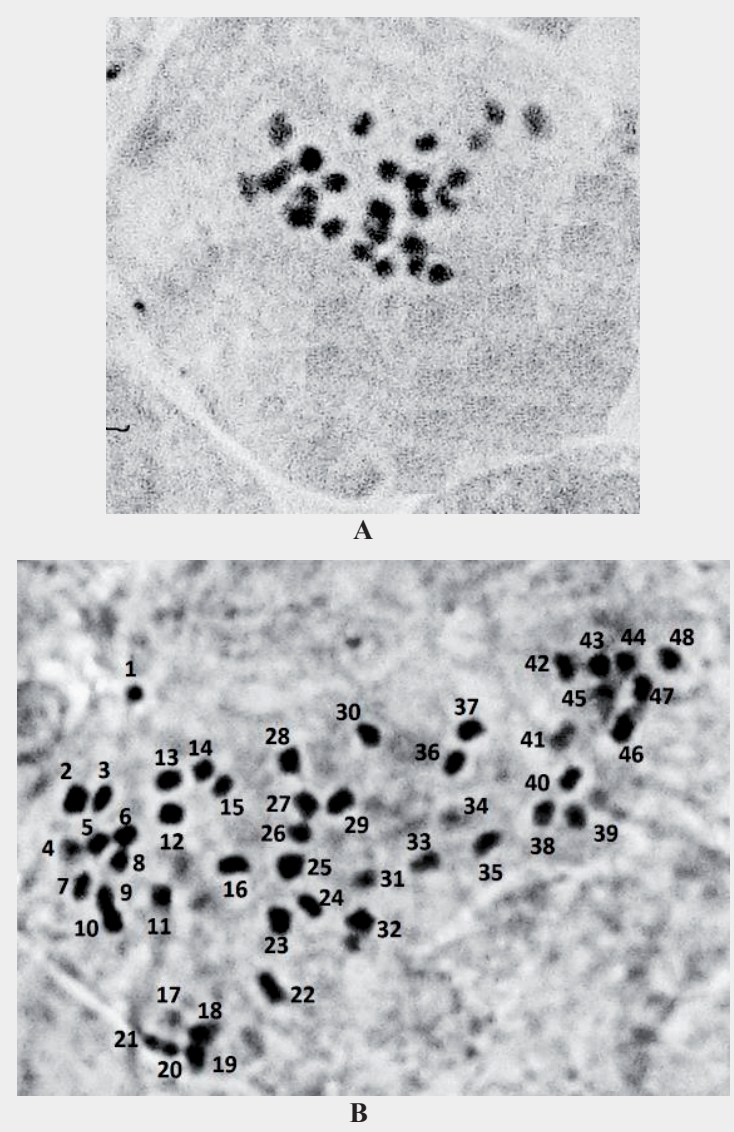

Figura 1. Número de cromosomas por célula somática. (A) Accesión 061979 con 24 cromosomas $(2 n=2 x=24)$. (B) Accesión 061445 de con 48 cromosomas $(2 n=4 x=48)$.
Julio - Dic. 2017 ISSN 0122-820X E-ISSN 2422-5053 
De acuerdo con la revisión taxonómica realizada por Spooner et al [11], las papas cultivadas se clasifican en cuatro especies, Solanum ajanhuiri, Solanum juzepczukii, Solanum curtilobum y Solanum tuberosum (con dos grupos Andigenum y Chilotanum). El grupo Andigenum incluye materiales diploide $(2 \mathrm{n}=2 \mathrm{x}=24)$, triploide $(2 \mathrm{n}=3 \mathrm{x}=36)$ y tetraploide $(2 \mathrm{n}=4 \mathrm{x}=48)$. Con base a esta clasificación, las accesiones identificadas como diploides y tetraploides dentro de la colección del banco de germoplasma continúan correspondiendo al grupo Andigenum. En estudios similares Uribe [12] encontró en papa criolla mayor número de los materiales diploide $(2 \mathrm{n}=2 \mathrm{x}=24)$. En ambos estudios hubo correspondencia del nivel de ploidía de los materiales analizados con la caracterización morfológica realizada posteriormente por Moreno y Valbuena [13].

No obstante Gaviria [14], en la determinación del nivel de ploidía y conteo de cromosomas en accesiones de papa chaucha triploide (anteriormente Solanum x chaucha, ahora $S$. tuberosum grupo Andigenum) de esta misma colección, reportó errores en la clasificación. Por lo tanto, se recomienda revisar esta colección estudiada, ya que algunas accesiones previamente clasificadas de acuerdo a caracteres morfológicos podrían estar catalogadas de forma incorrecta; esto repercutiría en la viabilidad de futuros cruzamientos para el mejoramiento genético de la especie. Cabe resaltar que el pequeño tamaño de los cromosomas o su solapamiento al observarlos al microscopio podría dificultar su observación y ser causantes de falsos positivos en la determinación de la dotación cromosómica de la especie de interés vegetal estudiada.

En cuatro montajes de dos accesiones se observaron células con 36 y 50 cromosomas (datos no mostrados). Estos resultados posiblemente se deben a artefactos de la técnica per se, sobre posición causada por la poca presión ejercida durante el squash, o a la pérdida de los cromosomas en el caso de células con 36 . Otra causa de las células con 36 cromosomas podría ser la eventual ruptura de la pared celular ocasionando la dispersión y mezcla de los cromosomas, quedando fuera y dentro del campo visual. En el caso de la célula con 50 cromosomas pudo haberse producido duplicación de algunos de ellos.

Algunos trabajos mencionan que es posible encontrar genotipos de papas que presentan raras aneuploidías. Mohanty et al. [15] identificaron alteraciones estructurales en cromosomas produciendo un fenómeno raro de aneugénesis $(2 \mathrm{n}=4 \mathrm{xC2}=50)$. Esta variación fue atribuida a la no disyunción mitótica de los tallos, dando origen a raíces aneusomáticas, sin embargo, se pudo confirmar que estas accesiones contaban con una dotación cromosómica de 48 y 24 cromosomas perteneciendo al grupo andigenum (Tabla II).

La técnica citogenética usada en esta investigación ha sido aplicada con éxito en diferentes especies vegetales, como tomate [16], banano [17], y pasto [18], para determinar el número cromosómico y nivel de plodía, sin embargo en la actualidad no se cuenta con mayor información acerca de la determinación de ploidía de la especie $S$. tuberosum L., y si existe no esta disponible. 
Tabla II. Número de cromosomas y número de cloroplastos por célula guarda de las 30 accesiones evaluadas.

\begin{tabular}{|c|c|c|c|c|}
\hline $\begin{array}{l}\text { CÓDIGO DE } \\
\text { COLECCIÓN }\end{array}$ & $\begin{array}{c}\text { NÚMERO DE } \\
\text { CROMOSOMAS }\end{array}$ & $\begin{array}{c}\text { NÚMERO DE } \\
\text { CLOROPLASTOS }\end{array}$ & PLOIDÍA & GRUPO \\
\hline 061291 & 48 & 12 & $* 2 n=4 x=48$ & Andigenum \\
\hline 061641 & 48 & 13 & $2 n=4 x=48$ & Andigenum \\
\hline 061951 & 48 & 12 & $2 n=4 x=48$ & Andigenum \\
\hline 062107 & 48 & 12 & $2 n=4 x=48$ & Andigenum \\
\hline 061300 & 48 & 14 & $2 n=4 x=48$ & Andigenum \\
\hline 061273 & 48 & 14 & $2 n=4 x=48$ & Andigenum \\
\hline 061777 & 48 & 14 & $2 n=4 x=48$ & Andigenum \\
\hline 061932 & 48 & 13 & $2 n=4 x=48$ & Andigenum \\
\hline 061286 & 48 & 13 & $2 \mathrm{n}=4 \mathrm{x}=48$ & Andigenum \\
\hline 060188 & 48 & 14 & $2 n=4 x=48$ & Andigenum \\
\hline 061336 & 48 & 12 & $2 n=4 x=48$ & Andigenum \\
\hline 061327 & 48 & 14 & $2 n=4 x=48$ & Andigenum \\
\hline 062039 & 48 & 13 & $2 n=4 x=48$ & Andigenum \\
\hline 062357 & 48 & 12 & $2 n=4 x=48$ & Andigenum \\
\hline 061815 & 48 & 14 & $2 n=4 x=48$ & Andigenum \\
\hline 061469 & 48 & 14 & $2 n=4 x=48$ & Andigenum \\
\hline 062138 & 48 & 14 & $2 n=4 x=48$ & Andigenum \\
\hline 061295 & 48 & 14 & $2 n=4 x=48$ & Andigenum \\
\hline 061439 & 48 & 14 & $2 n=4 x=48$ & Andigenum \\
\hline 061231 & 48 & 14 & $2 n=4 x=48$ & Andigenum \\
\hline 062071 & 48 & 13 & $2 n=4 x=48$ & Andigenum \\
\hline 061979 & 24 & 7 & $* * 2 n=2 x=24$ & Andigenum \\
\hline 061665 & 48 & 12 & $2 n=4 x=48$ & Andigenum \\
\hline 061445 & 48 & 14 & $2 n=4 x=48$ & Andigenum \\
\hline 061991 & 24 & 7 & $* * 2 n=2 x=24$ & Andigenum \\
\hline 061434 & 48 & 12 & $2 n=4 x=48$ & Andigenum \\
\hline 061632 & 48 & 12 & $2 n=4 x=48$ & Andigenum \\
\hline 062517 & 24 & 8 & $* * 2 n=2 x=24$ & Andigenum \\
\hline 062407 & 48 & 14 & $2 n=4 x=48$ & Andigenum \\
\hline 061415 & 48 & 14 & $2 n=4 x=48$ & Andigenum \\
\hline
\end{tabular}

\subsection{Conteo de cloroplastos en células guardas}

El número de cloroplastos por célula guarda en las accesiones 061979, 062517, 061991 varió entre 7 y 8 ; mientras que en las demás accesiones osciló entre 12 y 14, lo que indica su nivel diploide y tetraploide, respectivamente (Tabla II). Un ejemplo de lo observado en cada grupo se presenta en la Figura 2. Según Huamán [19] plantas con 7 y 8 cloroplastos por célula guarda, corresponden a diploides; mientras que plantas con 12 a 14 corresponden a tetraploides.

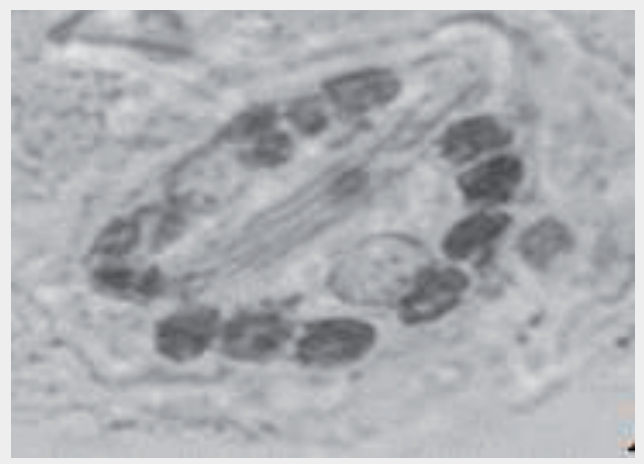

A

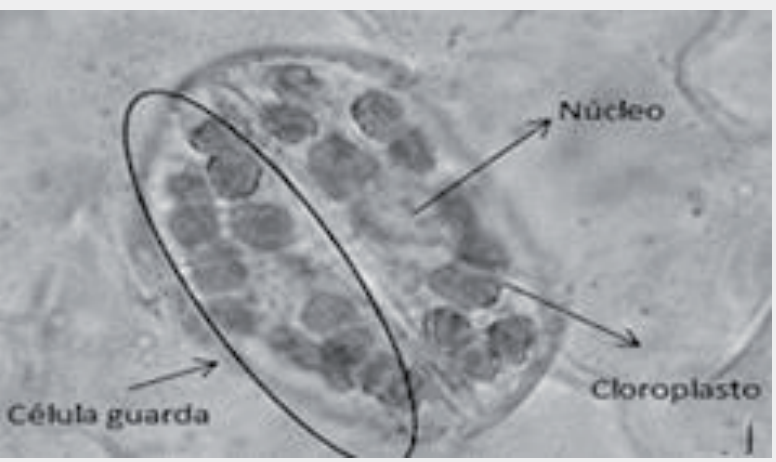

B

Figura 2. Número de cloroplastos por células guarda. (A) accesión 061445 con 7 cloroplastos.

(B) accesión 061979 con 12 cloroplastos.

Los dos procedimientos usados en la presente investigación, proporcionan información que confirma el nivel de ploidía del material en estudio. Si bien, el conteo del número de cloroplastos en las células guarda de los estomas, no es una técnica que determina con exactitud la ploidía de genotipos, sin embargo, cuando se trata de gran cantidad de materiales, permite discriminar rápidamente, el grupo diploide de otros grupos [9]. En esta investigación, los resultados son 
concordantes con lo indicado por Huamán [19]. El autor señala que el juego cromosómico en celulas somáticas, guarda correlación con el número de cloroplastos en las células guarda. Por otro lado, para determinar la ploidía con mayor precisión y exactitud se recomiendan técnicas como FISH, citometria de flujo y marcadores moleculares.

\section{Conclusiones}

En 30 accesiones de la colección de trabajo establecida en CORPOICA, se identificaron 27 accesiones con $2 n=4 x=48$ cromosomas y con 12 a 14 cloroplastos en las células guarda y tres accesiones tuvieron $2 \mathrm{n}=2 \mathrm{x}=$ 24 cromosomas con 7 a 8 cloroplastos en las células guarda.

El conteo del número de cloroplastos en células guarda, demuestra ser una técnica efectiva para discriminar material diploide y tetraploide, en primera aproximación, cuando se trata de colecciones con alto número de accesiones.

En esta investigación se confirma la clasificación taxonómica preliminar de los materiales estudiados como $S$. tuberosum grupo Andigenum, del mismo modo ha permitido discernir mejor los diferentes niveles de ploidía existentes en este grupo taxonómico.

\section{Agradecimientos}

12
Al Ministerio de Agricultura y Desarrollo Rural, Bogotá, por el apoyo económico para la realización de esta investigación. A María de Socorro, por el suministro del material vegetal de la colección que administra Corpoica. A Érika Sánchez, por su asistencia técnica. A V. Núñez, por su enseñanza y dirección del trabajo.

\section{Referencias}

[1] Organización de las Naciones Unidas para la Alimentación y la Agricultura, "Papas, patatas-Cultivos y producción", FAOSTAT database/Food and Agriculture Organization of the United Nations, 2014. [En línea]. Disponible en: http://faostat.fao.org/site/339/ default.aspx. [Accedido en: 14-ago2016]

[2] A. Ovchinnikova et al., "Taxonomy of cultivated potatoes (Solanum section Petota: Solanaceae)", Botanical Journal of the Linnean Society, vol. 165, no. 2, pp. 107-155, 2011.

[3] Federación Colombiana de la PapaFEDEPAPA, "Boletín Mensual Nacional", Fondo Nacional de Fondo de la Papa. [En línea]. Disponible en: http://fedepapa.com/wp-content/ uploads/2016/01/BOELTIN REGIONAL-NACIONAL.pdf. [Accedido: 10-sep-2016]

[4] C.E. Nústez, Variedades colombianas de papa. Bogotá: Universidad Nacional de Colombia, 2011.

[5] M. Mathias, B. Sagredo y J. Kalazich, "Uso de marcadores SSR para identificación de germoplasma de papa en el Programa de Mejoramiento de INIA de Chile", Agricultura Técnica, vol. 67, no. 1, 2007.

[6] R. Machida-Hirano, "Diversity of potato genetic resources", Breeding science, vol. 65, no 1, pp. 26-40, 2015.

[7] D. M. Spooner, M. Ghislain, R. Simon, S.H. Jansky and T. Gavrilenko, "Systematics, diversity, genetics, and evolution of wild and cultivated potatoes", The Botanical Review, vol. 80, no. 4, pp. 283-383, 2014. 
[8] R.A. Valencia y G. A. Ligarreto, "Estado del arte de los recursos genéticos vegetales en Colombia: Sistema de Bancos de Germoplasma", Revista Corpoica Ciencia y Tecnología Agropecuaria, vol. 11, no. 1, pp. 85-94, 2010.

[9] M. Orrillo y M. Bonierbale, Biología reproductiva y citogenética de la papa-Manual Técnico. Lima: Centro Internacional de la Papa (CIP), 2009.

[10] S. De Haan. J. Núñez, M. Bonierbale, M. Ghislain and J. Van der Maesen. "A Simple Sequence Repeat (SSR) marker comparison of a large in and exsitu potato landrace cultivar collection from Peru reaffirms the complementary nature of both conservation strategies", Diversity, vol.5, no. 3, pp. 505-521, 2013.

[11] D.M. Spooner, M. Ghislain. R. Simon, S. Jansky and T. Gavrilenko. "Systematics, diversity, genetics, and evolution of wild and cultivated potatoes". The Botanical Review, vol. 80, no. 4, pp. 283-383, 2014.

[12] F. Uribe, "Comprobación del nivel de ploidía en accesiones de papa criolla pertenecientes al banco de Germoplasma Vegetal de Corpoica", trabajo de fin de grado, Universidad de Cundinamarca, Facatativá, 2011.

[13] J.D. Moreno e I. Valbuena, "Colección Central Colombiana de Papa. La mayor riqueza de variabilidad genética para el mejoramiento del cultivo de papa en Colombia”. Corpoica, Bogotá, 2006.

[14] R. Gaviria, "Determinación del nivel de ploidía y conteo de cromosomas en accesiones de papa chaucha (Solanum $\mathrm{x}$ chaucha), procedentes del banco de germoplasma vegetal que administra
Corpoica", trabajo de fin de grado, Universidad de Cundinamarca, Facatativá, 2011.

[15] I.C. Mohanty, D. Mahapatra, S. Mohanty and A.B. Das, "Karyotype analyses and studies on the nuclear DNA content in 30 genotypes of potato (Solanum tuberosum L.)", Cell Biology International, vol. 28, no. 8-9, pp. 625633, 2004.

[16] F. Ramírez et al., "Caracterización de tetraploides y formación de híbridos triploides en tomate de cáscara", Ciencia $U A N L$, vol. 16 , no. 64, pp. 5566, 2013.

[17] H. Izquierdo, M.C. González y M. Núñez, "Estabilidad genética de las plantas de banano (Musa spp.) micropropagadas con reguladores del crecimiento no tradicionales", Biotecnología Aplicada, vol. 31, no. 1, pp. 18-22, 2014.

[18] S. Garduño et al., "Nivel de ploidía en poblaciones de Leptochloa dubia (Kunth) Nees nativas de México", Revista Mexicana de Ciencias Agrícolas, vol. 6, no. 3, pp. 539-548, 2015.

[19] Z. Huamán, Técnicas citológicas para determinar el número cromosómico y la fertilidad de las papas. Lima: Centro Internacional de la Papa (CIP), 1995. 